\title{
PERIODIC ORBITS FOR THE PLANAR NEWTONIAN THREE-BODY PROBLEM COMING FROM THE ELLIPTIC RESTRICTED THREE-BODY PROBLEMS
}

\author{
JAUME LLIBRE AND DONALD G. SAARI
}

\begin{abstract}
Through the introduction of a new coordinate system followed by a Poincare compactification, a new relationship is developed to connect the planar three-body problem with the various planar restricted three-body systems. This framework is further used to develop new conditions for the continuation of symmetric periodic orbits from elliptic restricted systems to the full planar threebody problem.
\end{abstract}

\section{INTRODUCTION}

The purpose of this paper is to study the relationship between the planar periodic orbits of the general Newtonian three-body problem and the planar periodic orbits that arise in different kinds of restricted three-body problems. We make two kinds of contributions. The first is to establish a new and sharper mathematical relationship between the restricted problems and the general three-body problems. We do this by using an appropriate coordinate system so that each of the different kinds of restricted problems (circular, elliptic, etc.) are on the "boundary" of the general three-body problem. Then we use this coordinate system to continue periodic orbits from any elliptic restricted three-body problem to the general three-body problem.

The derivation of $N$-body periodic orbits by continuing periodic orbits (symmetric or nonsymmetric) from the restricted to the general three-body problem is a classical theme that has been studied by several authors. In particular, we call attention to works of Poincaré [Po], Hadjidemetrious [Ha], and Meyer $[\mathrm{M} 1, \mathrm{M} 2]$ and the papers they reference. However, for the most part, most of the previous research concerning the continuation of periodic orbits impose strong conditions on the orbit of the restricted problem such as requiring them to be essentially circular or that one particle must be either very close to one of the primaries or very far from both primaries, etc. Our conditions hold for a wide class of orbits; it depends only on a certain property of the initial elliptic restricted three-body problem. To develop these ideas, we analyze the continuation of symmetric periodic orbits (Theorem 6.2). Our periodic orbits

Received by the editors April 27, 1994 and, in revised form, October 6, 1994.

1991 Mathematics Subject Classification. Primary 70F07; Secondary 70F15.

Key words and phrases. Periodic orbits, three-body problem, restricted problem.

J. Llibre acknowledges support from grant DGICYT \#PB90-0695; D. G. Saari acknowledges support from NSF IRI-9103180. 
are periodic in a convenient moving coordinate system; therefore, in general, these orbits are quasi-periodic in an inertial coordinate system.

\section{COORDINATES AND EQUATIONS OF MOTION OF THE THREE-BODY PROBLEM}

Let $P_{0}, P_{1}$ and $P_{2}$ be three particles of masses $m_{0}, m_{1}$ and $m_{2}$ respectively, moving in the plane $\mathbb{R}^{2}$ according to Newton's law of gravitation. We can assume that $M$, the center of mass of three bodies, is at the origin of an inertial coordinate system.

Our analysis involves using appropriate coordinates for the general threebody problem; coordinates that capture the interaction between the three-body and the restricted three-body problems. These coordinates come from three related coordinate systems. The first is the inertial system with $M$ at the origin and coordinates $(X, Y)$. The second is a moving coordinate system with coordinates $(u, v)$ that is defined in the following manner: the $u$-axis is the line passing through the particles $P_{0}$ and $P_{1}$ where the origin $O$ is at the center of mass of $m_{0}$ and $m_{1}$; see Figure 1 . The positive $u$-axis contains the particle $P_{1}$.

The third coordinate system, with coordinates $(x, y)$, is a moving coordinate system with origin $M$ where the $x$-axis is parallel to the $u$-axis from the moving coordinate system. The position of particle $P_{i}$ is given by $R_{i}=\left(X_{i}, Y_{i}\right)$, $R_{i}=\left(u_{i}, v_{i}\right)$ or $R_{i}=\left(x_{i}, y_{i}\right)$ depending on which of the three coordinate systems is being used. Let $\theta$ be the angle from the $X$-axis to $x$-axis.

The coordinates we use are a mixture of terms from these moving coordinate systems. To define these coordinates, note that the kinetic energy in the inertial system is given by

$$
T=\frac{1}{2} \sum_{i=0}^{2} m_{i}\left(\dot{X}_{i}^{2}+\dot{Y}_{i}^{2}\right) .
$$

Since $X_{i}=x_{i} \cos \theta-y_{i} \sin \theta$ and $Y_{i}=x_{i} \sin \theta+y_{i} \cos \theta$, we have that

$$
\dot{X}_{i}^{2}+\dot{Y}_{i}^{2}=\left(\dot{x}_{i}-\dot{\theta} y_{i}\right)^{2}+\left(\dot{y}_{i}+\dot{\theta} x_{i}\right)^{2}
$$

Let $r_{m}$ be the position vector of $M$ in the $(u, v)$ moving coordinate system. Since $O$ is the center of mass of $m_{0}$ and $m_{1}$, we have that $\left(m_{0}+m_{1}\right) r_{m}+$ $m_{0} R_{0}+m_{1} R_{1}=0$, or, from $m_{0} R_{0}+m_{1} R_{1}+m_{2} R_{2}=0$, that

$$
r_{m}=\left[m_{2} /\left(m_{0}+m_{1}\right)\right] R_{2} .
$$

We contend that since the system has only 4 degrees of freedom (the center of mass $M$ is fixed), the motion can be represented by the coordinates $\left(u_{1}, x_{2}, y_{2}, \theta\right)$. To see that this is the case, notice from $m_{0} u_{0}+m_{1} u_{1}=0$ that the position of $P_{0}$ is given by $u_{0}=-\left(m_{1} / m_{0}\right) u_{1}$. From $R_{0}=r_{0}-r_{m}$ and $R_{1}=r_{1}-r_{m}$ we have the remaining relationships

$$
x_{0}=-\frac{m_{1}}{m_{0}} u_{1}-\frac{m_{2}}{m_{0}+m_{1}} x_{2}, \quad y_{0}=-\frac{m_{2}}{m_{0}+m_{1}} y_{2} ;
$$

and

$$
x_{1}=u_{1}-\frac{m_{2}}{m_{0}+m_{1}} x_{2}, \quad y_{1}=-\frac{m_{2}}{m_{0}+m_{1}} y_{2}
$$




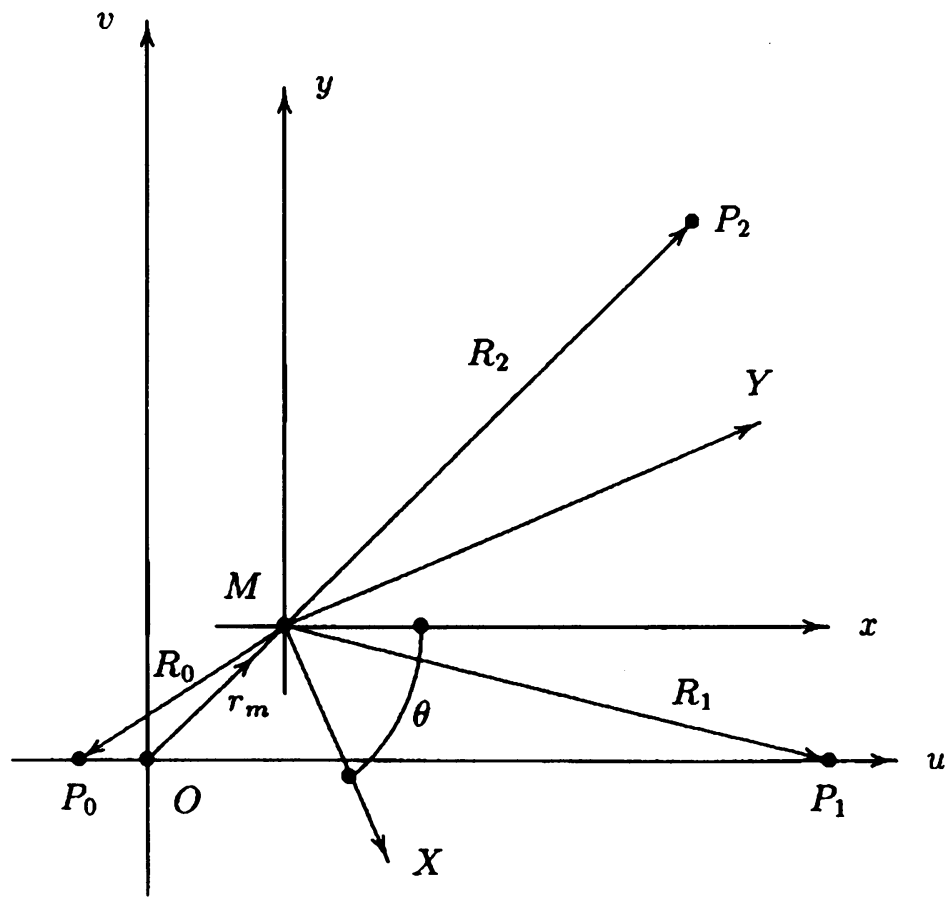

FIGURE 1. The coordinate systems

Therefore, making the above substitutions for $\left(x_{0}, y_{0}\right)$ and $\left(x_{1}, y_{1}\right)$ in (1) changes the kinetic energy to

$$
\begin{aligned}
T=\frac{1}{2}\left\{\frac{m_{1}\left(m_{0}+m_{1}\right)}{m_{0}}\left(\dot{u}_{1}^{2}+\dot{\theta}^{2} u_{1}^{2}\right)\right. \\
\left.+\frac{m_{2}\left(m_{0}+m_{1}+m_{2}\right)}{m_{0}+m_{1}}\left[\left(\dot{x}_{2}-\dot{\theta} y_{2}\right)^{2}+\left(\dot{y}_{2}+\dot{\theta} x_{2}\right)^{2}\right]\right\} .
\end{aligned}
$$

In what follows we use the coordinates $(s, x, y, \theta)=\left(u_{1}, x_{2}, y_{2}, \theta\right)$. Assume that the unit of length is chosen so that the gravitational constant equals unity. Then the potential energy is

$$
V=-\sum_{0 \leq i<j \leq 2} \frac{m_{i} m_{j}}{r_{i j}}
$$

where $r_{i j}$ denotes the distance between the particles $P_{i}$ and $P_{j}$. So

$$
\begin{aligned}
& r_{01}=\frac{m_{0}+m_{1}}{m_{0}} s, \\
& r_{02}^{2}=\left[\left(\frac{m_{2}}{m_{0}+m_{1}}+1\right) x+\frac{m_{1}}{m_{0}} s\right]^{2}+\left(\frac{m_{2}}{m_{0}+m_{1}}+1\right)^{2} y^{2}, \\
& r_{12}^{2}=\left[\left(\frac{m_{2}}{m_{0}+m_{1}}+1\right) x-s\right]^{2}+\left(\frac{m_{2}}{m_{0}+m_{1}}+1\right)^{2} y^{2} .
\end{aligned}
$$

Therefore the Lagrangean equations of motion

$$
\frac{d}{d t}\left(\frac{\partial T}{\partial \dot{q}}\right)=\frac{\partial L}{\partial q} \quad \text { for } q \in\{s, x, y, \theta\},
$$


associated to the Lagrangian $L=T-V$ are

$$
\begin{gathered}
\frac{d}{d t} \dot{s}=\dot{\theta}^{2} s-\frac{m_{0}}{m_{1}\left(m_{0}+m_{1}\right)} \frac{\partial V}{\partial s} \\
\frac{d}{d t}(\dot{x}-\dot{\theta} y)=\dot{\theta}(\dot{y}+\dot{\theta} x)-\frac{m_{0}+m_{1}}{m_{2}\left(m_{0}+m_{1}+m_{2}\right)} \frac{\partial V}{\partial x} \\
\frac{d}{d t}(\dot{y}+\dot{\theta} x)=-\dot{\theta}(\dot{x}-\dot{\theta} y)-\frac{m_{0}+m_{1}}{m_{2}\left(m_{0}+m_{1}+m_{2}\right)} \frac{\partial V}{\partial y}
\end{gathered}
$$

and

$$
\frac{d}{d t}\left(\frac{m_{1}\left(m_{0}+m_{1}\right)}{m_{0}} \dot{\theta} s^{2}+\frac{m_{2}\left(m_{0}+m_{1}+m_{2}\right)}{m_{0}+m_{1}}[-y(\dot{x}-\dot{\theta} y)+x(\dot{y}+\dot{\theta} x)]\right)=0 .
$$

This last equation leads to the first integral representing the total angular momentum

$$
c=\frac{m_{1}\left(m_{0}+m_{1}\right)}{m_{0}} \dot{\theta} s^{2}+\frac{m_{2}\left(m_{0}+m_{1}+m_{2}\right)}{m_{0}+m_{1}}[-y(\dot{x}-\dot{\theta} y)+x(\dot{y}+\dot{\theta} x)] .
$$

Of course, these equations of motion also have the first integral given by the total energy $H=T+V$.

It is well-known (and easy to check) that the equations of motion (2) of the three-body problem are invariant under the symmetry

$$
(s, x, y, \theta, t) \mapsto(s, x,-y,-\theta,-t) .
$$

Since the variable $\theta$ does not appear explicitly in system (2) and, from (3), the variable $\dot{\theta}$ can be expressed as a function of the variables $s, x, y, \dot{x}, \dot{y}$ and the constant $c$, in what follows we consider only the first three equations of (2) where in place of $\dot{\theta}$ its value from (3) is used. This reduction, essentially, corresponds to the elimination of the nodes.

\section{SYMMETRIC PERIODIC ORBITS OF THE THREE-BODY PROBLEM}

By using the symmetry (4) of the equations of motion, if $r(t)=(s(t), x(t)$, $y(t))$ is a solution of system $(2)$, then so is $r(-t)=(s(-t), x(-t),-y(-t))$. The geometrical interpretation of this symmetry is that if $r(t)$ is a solution curve of (2) in the configuration space $\mathbb{R}^{+} \times \mathbb{R}^{2}=\left\{(s, x, y) \in \mathbb{R}^{3}: s>0\right\}$, then its mirror image $r(-t)$ with respect to the $(s, x)$-plane (i.e., $y=0)$ is another solution where $-t$ replaces $t$.

This symmetry can be exploited, in a standard way, to obtain periodic orbits. Suppose that $r(t)$ passes through the $(s, x)$-plane at a point where the velocity is perpendicular to the $(s, x)$-plane, i.e. $\dot{s}=\dot{x}=0$. Using symmetry and the uniqueness of solutions, we have that $r(t)$ and its mirror image $r(-t)$ are the same solution. Now, should there be another time where the orbit $r(t)$ passes through the $(s, x)$-plane at a point where the velocity is perpendicular to the $(s, x)$-plane, then, due to the symmetry, the solution curve $r(t)$ is closed; i.e., the orbit $r(t)$ is periodic. In short, this proves the following standard result.

Proposition 3.1. Let $r(t)=(s(t), x(t), y(t))$ be a solution of the three-body problem (2). If $(y(t), \dot{s}(t), \dot{x}(t))$ is zero at $t=0$ and at $t=T / 2$ but at no other value of $t \in(0, T / 2)$, then $r(t)$ is a periodic solution of period $T$.

The periodic orbits given by Proposition 1 are called symmetric periodic orbits of the three-body problem. The symmetry conditions of the proposition 
require that at the two values $t=0, T / 2$ the three particles form a collinear configuration as the particle $m_{2}$ orthogonally crosses this line precisely when the distance between particles $m_{0}, m_{1}$ reaches a critical point $(\dot{s}(t)=0)$. If such a situation occurs, then the motion described by the three bodies in the rotating coordinate system is periodic of period $T$.

\section{RESTRICTED THREE-BODY PROBLEMS}

To obtain the restricted problems, we assume that the value of mass $m_{2}$ is infinitesimally small. This means that the gravitational force of $m_{2}$ on $m_{0}$ and $m_{1}$ is negligible, so the motion of the masses $m_{0}$ and $m_{1}$ is governed by the equations of the two-body problem. With an appropriate choice of the unit of mass, we have $m_{0}=1-\mu$ and $m_{1}=\mu, \mu \in(0,1)$, so system (2) becomes

$$
\begin{aligned}
\frac{d}{d t} \dot{s} & =\dot{\theta}^{2} s-\frac{(1-\mu)^{3}}{s^{2}}, \\
\frac{d}{d t}(\dot{x}-\dot{\theta} y) & =\dot{\theta}(\dot{y}+\dot{\theta} x)-\frac{(1-\mu)(x+(\mu s) /(1-\mu))}{r_{02}^{3}}-\frac{\mu(x-s)}{r_{12}^{3}}, \\
\frac{d}{d t}(\dot{y}+\dot{\theta} y) & =-\dot{\theta}(\dot{x}-\dot{\theta} y)-\frac{(1-\mu) y}{r_{02}^{3}}-\frac{\mu y}{r_{12}^{3}}
\end{aligned}
$$

where

$$
\begin{aligned}
\dot{\theta} & =\frac{(1-\mu) c}{\mu s^{2}}, \\
r_{02}^{2} & =\left(x+\frac{\mu}{1-\mu} s\right)^{2}+y^{2}, \\
r_{12}^{2} & =(x-s)^{2}+y^{2} .
\end{aligned}
$$

The assertion that the motion of the particles $m_{0}, m_{1}$ is independent of $m_{2}$ is reflected by the equation for $s$ in system (5); by using the equation for $\dot{\theta}$ it follows that the equation for $s$ becomes

$$
\ddot{s}=\left[\frac{(1-\mu) c}{\mu}\right]^{2} \frac{1}{s^{3}}-(1-\mu)^{3} \frac{1}{s^{2}} \quad(s>0) .
$$

This equation is related to the central force problem where $\mathbf{r}$ is the vector position of a particle. The equations of motion $\ddot{\mathbf{r}}=-m \mathbf{r} / r^{3}$ admit the integral $\mathbf{r} \times \frac{d}{d t} \mathbf{r}=\mathbf{C}$. So, if we let $r=|\mathbf{r}|$, then the equation of motion for the scalar $r$ become

$$
\ddot{r}=\frac{c^{2}}{r^{3}}-\frac{m}{r^{2}} \quad(r>0),
$$

where $c=|\mathbf{C}|$.

It now follows from the above that for every value of the total angular momentum $c$ and every solution $\tilde{s}(t)$ of system (6a), we have that system (5) defines a different restricted three-body problem; it can be a circular, elliptic, parabolic or hyperbolic restricted three-body problem depending on the nature of the solution $\tilde{s}(t)$.

The motion given by system (5) is called the motion of a restricted threebody problem because the two bodies of masses $m_{0}$ and $m_{1}$ (called primaries) 
move under the influence of their mutual gravitational attraction describing a solution of the two-body problem (6b), and the third body $m_{2}$ (attracted by the primaries but not influencing their motion) moves in the plane defined by the motion of the two primaries. To avoid singular situations, we now restrict attention to those settings where $c \neq 0$. The effect of this assumption is to exclude orbits with collisions between the two primaries.

Our discussion of the continuation of solutions requires describing the phase portrait of equation (6). So, toward this end, if we let

$$
w=\dot{s}, \quad a=\left[\frac{(1-\mu) c}{\mu}\right]^{2}, \quad b=(1-\mu)^{3},
$$

then the differential equation (6) becomes

$$
\dot{s}=w, \quad \dot{w}=a \frac{1}{s^{3}}-b \frac{1}{s^{2}} .
$$

This equation, of course, has physical meaning only when $s>0$. Our first goal is to study the compactified global phase portrait of this system first by adding the infinity boundary and then by analytically extending the flow to the enlarged space. With the change of the independent variable time, $d t=s^{3} \tau$, we obtain the polynomial system

$$
s^{\prime}=s^{3} w=P(s, w), \quad w^{\prime}=a-b s=Q(s, w),
$$

where the prime denotes the derivative with respect the new time $\tau$.

Let $X=(P, Q)$ be our polynomial vector field. Let $d$ be the degree of $X$, i.e., $d$ is the maximum degree of $P$ and $Q$; in our case $d=4$. To study the behavior of $X$ in a neighborhood of infinity, we use the Poincaré compactification (see for instance [Go] or [So]).

The idea of Poincare is the following. First, consider $\mathbb{R}^{2}$ imbedded in $\mathbb{R}^{3}$ in such a way that if $y=\left(y_{1}, y_{2}, y_{3}\right)$ represents an arbitrary point in $\mathbb{R}^{3}$, then $\mathbb{R}^{2}=\left\{y \in \mathbb{R}^{3}: y_{3}=1\right\}$. Then consider the sphere $\mathbb{S}^{2}=\left\{y \in \mathbb{R}^{3}: y_{1}^{2}+y_{2}^{2}+y_{3}^{2}=\right.$ 1) (the Poincaré sphere), which is tangent to $\mathbb{R}^{2}$ at the north pole $(0,0,1)$. Next, project the vector field $X$ in $\mathbb{R}^{2}$ into $\mathbb{S}^{2}$ by means of the central projections $p^{+}: \mathbb{R}^{2} \rightarrow \mathbb{S}^{2}$ and $p^{-}: \mathbb{R}^{2} \rightarrow \mathbb{S}^{2}$. That is, $p^{+}(y)$ (respectively $p^{-}(y)$ ) is the intersection of the line joining $y$ to the origin with the northern (respectively southern) hemisphere of $\mathbb{S}^{2}$. In this manner, an induced vector field, a copy of $X$, is obtained in both hemispheres.

Notice that the points at infinity in $\mathbb{R}^{2}$ (two for each direction) are in a one-to-one correspondence with the points of the equator, $\mathbb{S}^{1}=\left\{y \in \mathbb{S}^{2}: y_{3}=\right.$ $0\}$. If we attempt to extend the induced vector field on $\mathbb{S}^{2} \backslash \mathbb{S}^{1}$ to $\mathbb{S}^{2}$, we encounter difficulties as the vector field blows up as we approach $\mathbb{S}^{1}$. To avoid this complication, first multiply the vector field by the multiple $y_{3}^{d-1}$ at each $y \in \mathbb{S}^{2} \backslash \mathbb{S}^{1}$; an extension now becomes possible. Notice that for the extended field $p(X)$, the Poincaré compactification of $X, \mathbb{S}^{1}$ is an invariant set.

To obtain an analytic expression for $p(X)$, we use six local charts for the sphere $\mathbb{S}^{2}$ (considered as a differential manifold) given by $U_{k}=\left\{y \in \mathbb{S}^{2}: y_{k}>\right.$ $0\}, V_{k}=\left\{y \in \mathbb{S}^{2}: y_{k}<0\right\}$ for $k=1,2,3$. The corresponding coordinate maps $F_{k}: U_{k} \rightarrow \mathbb{R}^{2}$ and $G_{k}: V_{k} \rightarrow \mathbb{R}^{2}$, are defined by $F_{k}(y)=G_{k}(y)=$ $\left(y_{m} y_{k}^{-1}, y_{n} y_{k}^{-1}\right)$ for $m<n$ and $m, n \neq k$. Let $z=\left(z_{1}, z_{2}\right)$ denote the value of $F_{k}(y)$ or $G_{k}(y)$ for any $k$. Although the interpretation of $z$ depends upon 
which local chart is being used, there should be no confusion in what follows. Also note that the points of $\mathbb{S}^{1}$ in any local chart have $z_{2}=0$.

The expression of $p(X)$ in the local chart $\left(U_{1}, F_{1}\right)$ is given by

$$
\left(z_{2}^{d} m(z)\left[-z_{1} P\left(\frac{1}{z_{2}}, \frac{z_{1}}{z_{2}}\right)+Q\left(\frac{1}{z_{2}}, \frac{z_{1}}{z_{2}}\right)\right],-z_{2}^{d+1} m(z) P\left(\frac{1}{z_{2}}, \frac{z_{1}}{z_{2}}\right)\right),
$$

where $m(z)=\left(1+z_{1}^{2}+z_{2}^{2}\right)^{(1-d) / 2}$. The expression for $\left(U_{2}, F_{2}\right)$ and $\left(U_{3}, F_{3}\right)$ are

$$
\left(z_{2}^{d} m(z)\left[P\left(\frac{z_{1}}{z_{2}}, \frac{1}{z_{2}}\right)-z_{1} Q\left(\frac{z_{1}}{z_{2}}, \frac{1}{z_{2}}\right)\right],-z_{2}^{d+1} m(z) Q\left(\frac{z_{1}}{z_{2}}, \frac{1}{z_{2}}\right)\right),
$$

and $\left(m(z) P\left(z_{1}, z_{2}\right), m(z) Q\left(z_{1}, z_{2}\right)\right)$, respectively. For $k=1,2,3$, the expression for $p(X)$ in $\left(V_{k}, G_{k}\right)$ is the same that in $\left(U_{k}, F_{k}\right)$ multiplied by $(-1)^{d-1}$.

The expression for $p(X)$ in the different local charts is a polynomial vector field multiplied by a nonvanishing analytic factor. In what follows, we omit the factor $m(z)$ (this represents a simple scaling of the independent variable).

For notation, call the critical points of $p(X)$ in $\mathbb{S}^{2} \backslash \mathbb{S}^{1}$ (respectively $\mathbb{S}^{1}$ ) the finite (respectively infinite) critical points of $X$ of $p(X)$. The integral curves in $S^{2}$ are symmetric about the origin, so the flow of $p(X)$ is usually represented only on a closed hemisphere, either the northern or the southern. Either of these closed hemispheres is called the Poincaré disk.

In the local chart $U_{1}$ the vector field $p(X)$ is

$$
\left(-z_{1}^{2}-b z_{2}^{3}+a z_{2}^{4},-z_{1} z_{2}\right)
$$

with a unique infinite critical point at $(0,0)$; this is a degenerate critical point with an identically zero Jacobian matrix. So the local phase portrait at $(0,0)$ is studied by using the standard technique of blow-ups (see for instance [AI] or $[\mathrm{ALGM}])$. After a change of coordinates $\left(z_{1}, z_{2}\right) \mapsto\left(z_{3}, z_{2}\right)$ where $z_{3}=$ $z_{1} / z_{2}$, the vector field $\left.p(X)\right|_{U_{1}}$ becomes

$$
\left(-b z_{2}^{2}+a z_{2}^{3},-z_{2}^{2} z_{3}\right)
$$

To convert this into a linear vector field, using the scaling $z_{2}^{2}$ in the time variable. The resulting vector field

$$
\left(-b+a z_{2},-z_{3}\right)
$$

has a center at $(0, b / a)$. So the flow of the vector field $\left(-b z_{2}^{2}+a z_{2}^{3},-z_{2}^{2} z_{3}\right)$ is qualitatively represented by Figure 2. Consequently the flow of $\left.p(X)\right|_{U_{1}}$ is like that given in Figure 3.

The vector field $p(X)$ in the local chart $U_{2}$ is given by

$$
\left(z_{1}^{3}-a z_{1} z_{4}^{4}+b z_{1}^{2} z_{2}^{3}, b z_{1} z_{2}^{4}-a z_{2}^{5}\right) \text {. }
$$

The unique infinite critical point again is the degenerate critical point $(0,0)$ with identically zero Jacobian matrix. With the blow-up $\left(z_{1}, z_{2}\right) \mapsto\left(z_{1}, z_{3}\right)$ defined by $z_{3}=z_{2} / z_{1},\left.p(X)\right|_{U_{2}}$ becomes

$$
\left(z_{1}^{3}-a z_{1}^{5} z_{3}^{4}+b z_{1}^{5} z_{3}^{3},-z_{1}^{2} z_{3}\right)
$$

Using the scaling $z_{1}^{2}$ with the time variable, the vector field becomes

$$
\left(z_{1}-a z_{1}^{3} z_{3}^{4}+b z_{1}^{3} z_{3}^{3},-z_{3}\right) \text {. }
$$




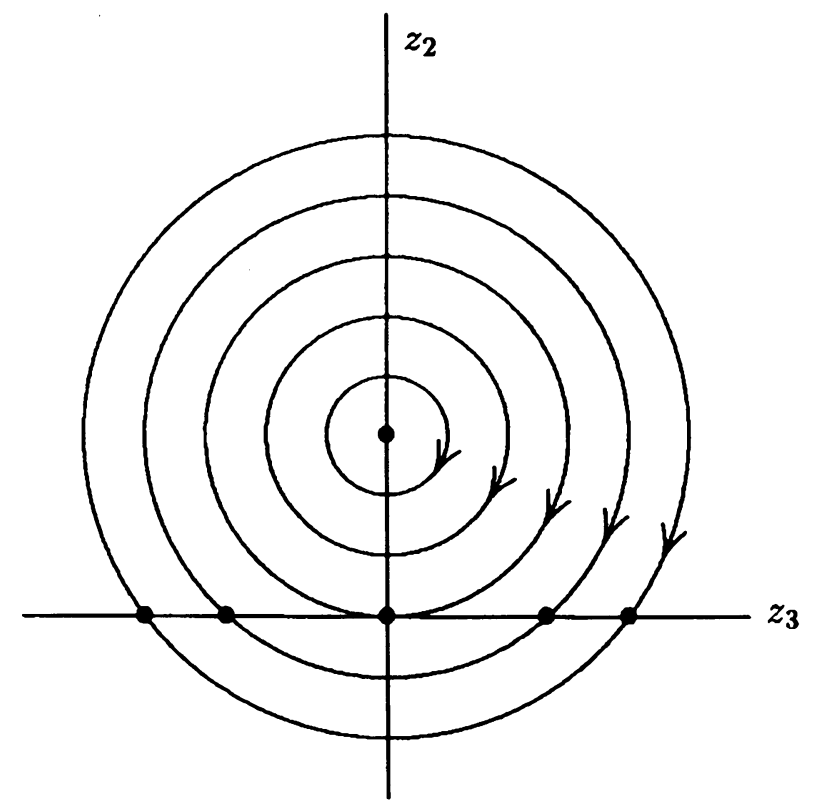

FIGURE 2. Flow of the vector field

The unique critical point on the line $z_{1}=0$ is $(0,0)$, an unstable saddle. Returning to the coordinates $\left(z_{1}, z_{2}\right)$ and recognizing that the axis $z_{1}=0$ and $z_{2}=0$ are invariant with respect to the vector field $\left.p(X)\right|_{U_{2}}$, it follows that the origin of $\left.p(X)\right|_{U_{2}}$ is a saddle, where the $z_{1}$-axis is formed by the unstable manifolds (or unstable separtrices) and the $z_{2}$-axis by the stable ones.

In the summary the global phase portrait of $p(X)$ in the Poincare disk is represented in Figure 4. But since equation (6) has a physical meaning only when $s>0$, we are only interested on the right half-phase portrait of the

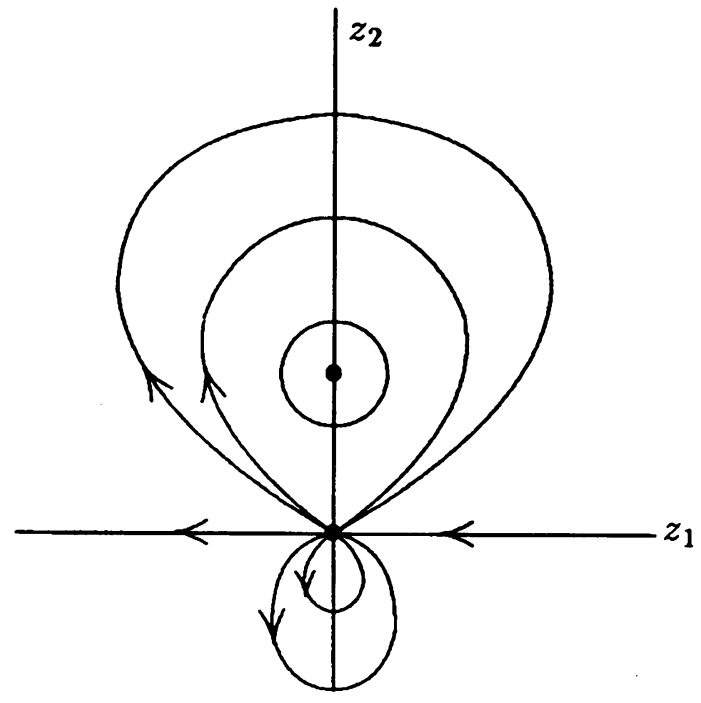

FigURE 3. Flow of $\left.p(X)\right|_{U_{1}}$ 


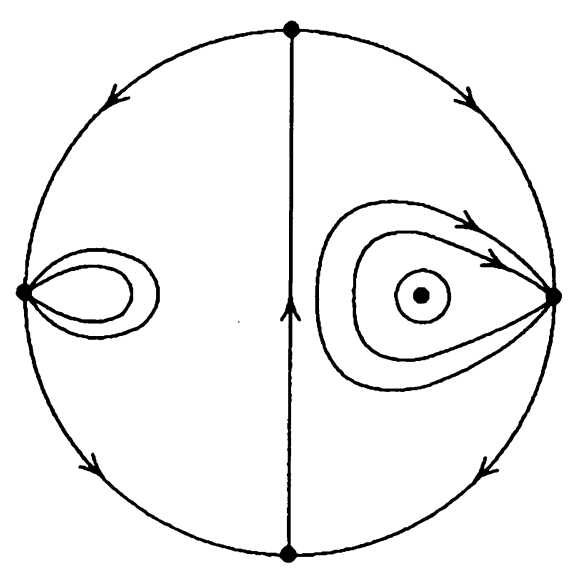

Figure 4. Poincaré disk

Poincare disk. Recall that $s$ is the distance from the center of mass of $1-\mu$ and $\mu$ to $\mu$, so it describes the radial motion between the two primaries $1-\mu$ and $\mu$ of the restricted three-body problem (because $m_{2}=0$ ).

Now we describe the different kinds of restricted three-body problems that we can obtain on the boundary of the three-body problem for a given value $c$ of the total angular momentum. Denote by

$$
h=\frac{1}{2} \dot{s}^{2}+\frac{a}{2 s^{2}}-\frac{b}{s}
$$

the first integral of system (6a). The assumption $c \neq 0$ ensures that $a>0$, so, for $h=-b^{2} /(2 a)$ the solution $\tilde{s}(t)$ of system (6) is the critical point (a center). Substituting this solution in system (5) we obtain a circular restricted three-body problem. If $-b^{2} /(2 a)<h<0$, then the solution $\tilde{s}(t)$ of system (6) is a periodic orbit. Therefore, this solution inside system (5) gives an elliptic restricted three-body problem. For $h=0$ the solution $\tilde{s}(t)$ of system (6) is a loop of the infinity critical point at the origin of $U_{1}$. Substituting this homoclinic orbit in system (5) we obtain a parabolic restricted three-body problem. Finally, if $h>0$, then the solution $\tilde{s}(t)$ of $(6)$ is a solution which starts and ends in the parabolic sectors of the infinity point at the origin of $U_{1}$. Therefore, this solution inside system (5) gives a hyperbolic restricted three-body problem.

Notice that only for the circular restricted three-body problem do we have that $\dot{\theta}$ is constant; here system (5) is autonomous. In the general case, $\dot{\theta}$ is given by the expression in system (5), and the equations of motion for the elliptic, parabolic and hyperbolic restricted three-body problems are nonautonomous.

\section{SYMMETRIC PERIODIC ORBITS OF A RESTRICTED THREE-BODY PROBLEM}

If we choose for $\tilde{s}(t)$ the solution given by the critical point or by a periodic solution of equation (6a), then we have that the motion described by system (5) corresponds to the circular or elliptic restricted three-body problem respectively. The equations of motion of such restricted problems have periodic orbits where some of them are invariant under the symmetry

$$
(x, y, t) \mapsto(x,-y,-t) .
$$


Using the same argument given in Section 3 we have for these restricted problems that:

Proposition 5.1. Let $r(t)=(x(t), y(t))$ be a solution of the circular or elliptic restricted three-body problem. If at $t=0$ and at $t=T / 2$ we have that $\dot{s}(t), \dot{x}(t)$ are $y(t)$ are zero, but not all three can be simultaneously zero for any $t \in$ $(0, T / 2)$, then $r(t)$ is a periodic solution of period $T$.

This proposition admits an interpretation similar to that of Proposition 3.1. Namely, at $t=0, T / 2$ the three particles form a collinear configuration when the infinitesimal body $m_{2}$ crosses perpendicularly the $x$-axis. Moreover, at these times, the distance between the primaries is at a critical point-for the elliptic problem, this means they are either at their closest or farthest approach. If these conditions do not occur at any other time in $(0, T / 2)$, the motion described by the three bodies in the moving coordinate system is periodic of period $T$. These periodic orbits are called symmetric periodic solutions of period $T$ for the circular or elliptic restricted three-body problem. Notice that for the elliptical restricted problem, the value of $T$ and the period of the primaries must be rationally related. No such constraint occurs for the circular restricted problem as $\dot{s}(t)=0$ for all time. While the degeneracy associated with the circular restricted problem makes it easier to find periodic orbits with numerical techniques, it also creates difficulties in the analysis of the continuation of these periodic orbits into the general three-body problem.

\section{CONTINUATION OF SYMMETRIC PERIODIC ORBITS}

In this section by using the method of analytic continuation (due to Poincare [Po]) with respect to the variable $m_{2}$, we derive a new sufficient condition for symmetric periodic orbits of the elliptic restricted three-body problem $\left(m_{2}=0\right)$ to be extended to symmetric periodic orbits (in our moving coordinate system) of the three-body problem for $m_{2}>0$ sufficiently small. An interesting fact is that this extension to $m_{2}>0$ depends only on a particular property of the elliptic restricted three-body problem.

Because of our choice of a coordinate system and because of Proposition 3.1, a symmetric periodic solution for the three-body problem exists if it can be shown that there exist initial conditions

$$
y=0, \quad \dot{s}=0, \quad \dot{x}=0 \quad \text { at } t=0,
$$

with the property that

$$
y=0, \quad \dot{s}=0, \quad \dot{x}=0 \quad \text { at } t=T / 2 .
$$

To show this, we use the method of analytic continuation; a method based on treating (7) as a system of equations where $m_{2}$ is one of the variables and where the properties of the solution at $t=T / 2$ are obtained by use of the implicit function theorem. In other words, start with $m_{2}=0$ and a symmetric periodic solution of the relevant restricted three-body problem. Then, appropriate conditions are derived so that the implicit function theorem can be used to ensure that (7) holds for solutions with $m_{2}>0$. So, let $\tilde{s}(t)$ be the solution which defines the restricted three-body problem for a fixed value $c$ of the total angular momentum through system (5). We can assume (by use of a time translate if needed) that $\dot{\tilde{s}}(0)=0$. 
Given initial values

$\mu \in(0,1), \quad s_{0}=\tilde{s}(0), \quad x_{0}, \quad y_{0}=0, \quad \dot{s}_{0}=0, \quad \dot{x}_{0}=0, \quad \dot{y}_{0}, \quad m_{2}=0$,

and sufficiently small values of

$$
\delta s_{0}, \quad \delta x_{0}, \quad \delta \dot{y}_{0}, \quad m_{2},
$$

the solution $(s(t), x(t), y(t))$ of the three-body problem (2) with initial conditions

$$
\begin{array}{lll}
s(0)=s_{0}+\delta s_{0}, & x(0)=x_{0}+\delta x_{0}, & y(0)=0, \\
\dot{s}(0)=0, & \dot{x}(0)=0, & \dot{y}(0)=y_{0}+\delta y_{0},
\end{array}
$$

can be developed in power series of $\delta s_{0}, \delta x_{0}, \delta \dot{y}_{0}$ and $m_{2}$ where the coefficients are functions of $t$ (see Poincare [Po]). This is given by

$$
\begin{aligned}
& s(t)=s_{1}(t)+O\left(m_{2}\right)+O_{2}, \\
& x(t)=x_{1}(t)+O\left(m_{2}\right)+O_{2}, \\
& y(t)=y_{1}(t)+O\left(m_{2}\right)+O_{2},
\end{aligned}
$$

where $s_{1}(t)=s_{1}\left(t ; s_{0}+\delta s_{0}\right)$ is the solution of

$$
\ddot{s}_{1}=\left[\frac{(1-\mu) c}{\mu}\right]^{2} \frac{1}{s_{1}^{3}}-(1-\mu)^{3} \frac{1}{s_{1}^{2}},
$$

satisfying $s_{1}(0)=s_{0}+\delta s_{0}$ and $\dot{s}_{1}(0)=0$; and

$$
\begin{aligned}
&\left(x_{1}(t), y_{1}(t)\right)=\left(x\left(t ; s_{0}+\delta s_{0}, x_{0}+\delta x_{0}, \dot{y}_{0}+\delta \dot{y}_{0}\right),\right. \\
&\left.y\left(t ; s_{0}+\delta s_{0}, x_{0}+\delta x_{0}, \dot{y}_{0}+\delta \dot{y}_{0}\right)\right)
\end{aligned}
$$

is the solution of

$$
\begin{aligned}
& \frac{d}{d t}(\dot{x}-\dot{\theta} y)=\dot{\theta}(\dot{y}+\dot{\theta} x)-\frac{(1-\mu)\left(x+\left(\mu s_{1}\right) /(1-\mu)\right)}{r_{02}^{3}}-\frac{\mu\left(x-s_{1}\right)}{r_{12}^{3}}, \\
& \frac{d}{d t}(\dot{y}+\dot{\theta} y)=-\dot{\theta}(\dot{x}-\dot{\theta} y)-\frac{(1-\mu) y}{r_{02}^{3}}-\frac{\mu y}{r_{12}^{3}}
\end{aligned}
$$

where

$$
\begin{gathered}
\dot{\theta}=\frac{(1-\mu) c}{\mu s_{1}^{2}}, \quad r_{02}^{2}=\left(x+\frac{\mu}{1-\mu} s_{1}\right)^{2}+y^{2}, \\
r_{12}^{2}=\left(x-s_{1}\right)^{2}+y^{2},
\end{gathered}
$$

and the solutions satisfy $x_{1}(0)=x_{0}+\delta x_{0}, y_{1}(0)=0, \dot{x}_{1}(0)=0$ and $\dot{y}_{1}(0)=$ $\dot{y}_{0}+\delta \dot{y}_{0}$.

In these equations, $O\left(m_{2}\right)$ represents terms of at least order 1 in the variable $m_{2}$; and $\mathrm{O}_{2}$ represents terms of at least order 2 in the variables $\delta s_{0}, \delta x_{0}$ and $\delta \dot{y}_{0}$.

Notice that the solution $s_{1}(t)$ is independent on $x_{0}+\delta x_{0}$ and $\dot{y}_{0}+\delta \dot{y}_{0}$ and that the solution $\left(x_{1}(t), y_{1}(t)\right)$ only depends on $s_{0}+\delta s_{0}$ through $\dot{\theta}$.

Now for $m_{2}=0$, suppose that the initial conditions

$$
s=s_{0}=\tilde{s}(0), \quad x=x_{0}, \quad y=0, \quad \dot{s}=0, \quad \dot{x}=0, \quad \dot{y}=\dot{y}_{0} \quad \text { at } t=0
$$


correspond to a symmetric periodic solution of period $T$ for the circular or elliptic restricted three-body problem. According to (7), we seek a solution $(s(t)$, $x(t), y(t))$ of (2) with sufficiently small values of $m_{2}>0$ so that

and

$$
\begin{array}{lll}
\dot{s}=\bar{s}=s_{0}+\delta s_{0}, & x=\bar{x}=x_{0}+\delta x_{0}, & y=0, \\
\dot{s}=0, \quad \dot{x}=0, & \dot{y}=\dot{\bar{y}}=\dot{y}_{0}+\delta \dot{y}_{0} & \text { at } t=0,
\end{array}
$$

Since the initial conditions (12) are close to the initial conditions (11) of the symmetric periodic orbit for $m_{2}=0$ and since the symmetric orbit crosses the $(s, x)$-plane perpendicularly, we have that the orbit with initial conditions (12) must also cross the $(s, x)$-plane $(y=0)$ transversally after a time $\bar{t}$ close to the half period of the symmetric periodic solution given by (11) when $m_{2}=$ 0 . Therefore, using the implicit function theorem to show the existence of the solution specified by the conditions (12) and (13), it is sufficient that the determinant

$$
\operatorname{det}\left(\frac{\partial(\dot{s}, \dot{x})}{\partial(\bar{s}, \bar{x})}\right)
$$

evaluated at $t=T / 2, \bar{s}=s_{0}, \bar{x}=x_{0}$ and $m_{2}=0$ is nonzero. When this is the case, the symmetric periodic solution given by (11) of the circular or elliptic restricted three-body problem can be continued to a symmetric periodic solution of the three-body problem (2) for sufficiently small values of $m_{2}>0$.

From (8), in the computation of (14), we can take $\dot{s}(t)=\dot{s}_{1}(t)$ and $\dot{x}(t)=$ $\dot{x}_{1}(t)$. A further reduction occurs because $\partial \dot{s}_{1} / \partial \bar{x}=0$; therefore the condition becomes

$$
\operatorname{det}\left(\frac{\partial\left(\dot{s}_{1}, \dot{x}_{1}\right)}{\partial(\bar{s}, \bar{x})}\right)=\frac{\partial \dot{s}_{1}}{\partial \bar{s}} \cdot \frac{\partial \dot{x}_{1}}{\partial \bar{x}}
$$

As a final reduction, we now prove that $\partial \dot{s}_{1} / \partial \bar{s}$ evaluated at $t=T / 2$ and $\bar{s}=s_{0}$ is nonzero for the elliptic restricted three-body problem and zero for the circular one. To show the first we shall use a theorem of Diliberto [Di] on the integration of the homogeneous variational equations of a plane autonomous differential equation in terms of geometric quantities along a given trajectory of the system. (Also see the paper by Chicone [Ch] where, in addition to using the Delibert Theorem to address his problem, he corrects a flaw in the theorem. We use Chicone's version.) Here we denote by $X=\left(X_{1}, X_{2}\right)$ a smooth plane vector field with flow $\phi_{t}$. The geometric quantities are the curvature, the curl and the divergence given by

$$
\begin{gathered}
\kappa=\|X\|^{-3}\left(X_{1} \dot{X}_{2}-X_{2} \dot{X}_{1}\right), \quad \operatorname{curl} X=\frac{\partial X_{2}}{\partial x_{1}}-\frac{\partial X_{1}}{\partial x_{2}}, \\
\operatorname{div} X=\frac{\partial X_{1}}{\partial x_{1}}+\frac{\partial X_{2}}{\partial x_{2}},
\end{gathered}
$$

where $\|X\|=\sqrt{\langle X, X\rangle}$ denotes the euclidean norm. The orthogonal vector field of $X$ is defined as $X^{\perp}=\left(-X_{2}, X_{1}\right)$. We also need the vector field $u_{X^{\perp}}$ parallel to $X^{\perp}$ given by

$$
u_{X^{\perp}}(x)=\frac{1}{\|X(x)\|^{2}} X^{\perp}(x) .
$$

The result of Diliberto can be stated as follows. 
Theorem 6.1. If $X(x) \neq 0$, then the linear variational equation along the integral curve $t \mapsto \phi_{t}(x)$,

$$
\dot{V}=D X\left(\phi_{t}(x)\right) V,
$$

has a fundamental matrix solution $\Phi(t)$, satisfying $\operatorname{det}(\Phi(0))=1$, given by

$$
\Phi(t)=\left[X\left(\phi_{t}(x)\right), V(t)\right],
$$

where

$$
V(t)=\alpha(t) X\left(\phi_{t}(x)\right)+\beta(t) u_{X^{\perp}}\left(\phi_{t}(x)\right)
$$

and

$$
\begin{aligned}
& \beta(t)=\beta(t ; X, x)=\exp \left(\int_{0}^{t} \operatorname{div} X\left(\phi_{s}(x)\right) d s\right) \\
& \alpha(t)=\alpha(t ; X, x)=\int_{0}^{t}\left\{\frac{1}{\|X\|^{2}}[2 \kappa\|X\|-\operatorname{curl} X]\right\}\left(\phi_{\tau}(x)\right) \beta(\tau) d \tau .
\end{aligned}
$$

The idea is to use Theorem 6.1 to compute $\partial \dot{s}_{1} / \partial \bar{s}$ for the elliptic restricted three-body problem. Consider the equation (9) in the form

$$
\dot{s}_{1}=v, \quad \dot{v}=\frac{a}{s_{1}^{3}}-\frac{b}{s_{1}^{2}},
$$

where $a=[(1-\mu) c / \mu]^{2}, b=(1-\mu)^{3}$. The linear variational equations are

$$
\frac{d}{d t}\left(\begin{array}{ll}
\frac{\partial s_{1}}{\partial \bar{s}} & \frac{\partial s_{1}}{\partial \bar{v}} \\
\frac{\partial \vec{v}}{\partial \bar{s}} & \frac{\partial v}{\partial \bar{v}}
\end{array}\right)=\left(\begin{array}{cc}
0 & 1 \\
\frac{2 b}{s_{1}^{3}}-\frac{3 a}{s_{1}^{4}} & 0
\end{array}\right)\left(\begin{array}{ll}
\frac{\partial s_{1}}{\partial \bar{s}} & \frac{\partial s_{1}}{\partial \bar{v}} \\
\frac{\partial v}{\partial \bar{s}} & \frac{\partial v}{\partial \bar{v}}
\end{array}\right)
$$

From Theorem 6.1, it follows that $\partial v / \partial \bar{s}$ is equal to $a s_{1}^{-3}-b s_{1}^{-2}$ the second component of our vector field $X=\left(\dot{s}_{1}, \dot{v}\right)$. Since $\partial s_{1} / \partial \bar{s}=\partial v / \partial \bar{s}$, it follows that

$$
\left.\frac{\partial \dot{s}_{1}}{\partial \bar{s}}\right|_{t=T / 2 ; \bar{s}=s_{0}}=a \tilde{s}\left(\frac{T}{2}\right)^{-3}-b \tilde{s}\left(\frac{T}{2}\right)^{-2},
$$

which is nonzero because we are considering the elliptic restricted three-body problem. Hence, from (15) it follows immediately that the continuation problem is reduced to computing one term. This is the following assertion:

Theorem 6.2. If the derivative $\partial \dot{x}_{1} / \partial \bar{x}$ evaluated at $t=T / 2, \bar{s}=\tilde{s}(0), \bar{x}=x_{0}$, $\dot{\bar{y}}=\dot{y}_{0}$ is nonzero, then the symmetric periodic solution with initial conditions (11) of the elliptic restricted three-body problem (5) with $s(t)=\tilde{s}(t)$ can be continued to the three-body problem (2) for values of $m_{2}>0$ sufficiently small.

Thus, Theorem 6.2 provides a sufficient condition depending only on the elliptic restricted three-body problem in order that its symmetric periodic solution can be continued to the general three-body problem.

We remark that for the circular restricted three-body problem we cannot use Diliberto's theorem to prove a similar result to Theorem 6.2 , because in the circular problem $X(\tilde{s}(t))=0$, and consequently the hypothesis of Theorem 6.1 is not satisfied. 


\section{REFERENCES}

[ALGM] A. A. Andronov, E. A. Leontovich, I. I. Gordon and A. L. Maier, Qualitative theory of second-order dynamic systems, Wiley, New York, 1973.

[AI] V. I. Arnold and Y. S. Ilyashenko, Dynamical systems I, Ordinary differential equations, Encyclopaedia of Math. Sci., vols. 1-2, Springer-Verlag, Heildelberg, 1988.

[Ch] C. Chicone, Bifurcations of nonlinear oscillations and frequency entrainment near resonance, SIAM J. Math. Anal. 23 (1992), 1577-1608.

[Di] S. I. Diliberto, On systems of ordinary differential equations, Contributions to the Theory of Nonlinear Oscillations, Ann. of Math. Stud., no. 20, Princeton Univ. Press, Princeton, NJ, 1950.

[Ha] J. D. Hadjidemetrious, The continuation of periodic orbits of the planar restricted to the general three-body problem, Celestial Mech. 12 (1975), 155-174.

[Go] E. A. V. Gonzales, Generic properties of polynomial vector fields at infinity, Trans. Amer. Math. Soc. 143 (1969), 201-222.

[M1] K. R. Meyer, Periodic solutions of the N-body problem, J. Differential Equations 39 (1981), 2-38.

[M2] , Apollonius coordinates, the $N$-body problem, and continuation of periodic solutions, J. Dynamics Differential Equation 3 (1991), 381-397.

[Po] H. Poincaré, Les mèthodes nouvelles de la mécanique céleste, 3 volumes, Gauthier-Villars, Paris, 1892-1899; reprinted by Dover,New York, 1957.

[So] J. Sotomayor, Curvas definidas por equacẽs diferenciais no plano, Instituto de Matemética Pura e Aplicada, Rio de Janeiro, 1981.

Department of Mathematics, Northwestern University, 2033 Sheridan Road, EVANSTON, ILLINOIS 60208

E-mail address: d-saari@math.nwu.edu

Department de Matemàtiques, Universitat Autònoma de Barcelona, Bellaterra, 08193 Barcelona, SPAIN

E-mail address: imat0@cc.uab.es 